\title{
Liver Hamartomas in Patients on Oral Contraceptives
}

\author{
J. P. O'SULLIVAN, R. P. WILDING
}

British Medical fournal, 1974, 3, 7-10

\section{Summary}

Three cases are reported of tumour-like lesions of the liver in women who had been on oral contraceptives for long periods. These malformations have a prominent vascular component and may present with haemoperitoneum and shock. The possibility of an association between liver hamartomas and oral contraceptive therapy has to be considered.

\section{Introduction}

Since October 1973, 11 cases of benign liver cell tumour have been described in women who had been on oral contraceptives. We report three similar cases which have been referred to this hospital in the past four years. These are the first cases to be reported from the United Kingdom in which there was a possible association between oral contraceptive therapy and the development of liver cell nodules.

\section{Case Reports}

Case 1.-A 34-year-old woman had taken oral contraceptives for six years with two 12-month gaps when she had her fourth and fifth children. The agents used were lynoestrenol-mestranol (Lyndiol), ethynodiol-mestranol (Ovulen), and norethisteronemestranol (Gynovlar 21). She presented with dizziness, headaches, and substernal pain of three months' duration. Recently she had noticed loose motions. A mass was felt in the right hypochondrium and at laparotomy (July 1970) a pale yellow solitary tumour was found in the right lobe of the liver. The tumour had a nodular surface and a biopsy specimen was taken but the histological report was inconclusive. Tubal ligation was also performed. Postoperatively arteriography showed the tumour to contain an abnormal vasculature. She was then referred to our unit and on 14 August 1970 local resection of the tumour was performed by segmental excision of the tip of the right lobe of the liver. She

made an uninterrupted recovery and had remained well almost four years later.

Case 2.-A 35-year-old woman had taken oral contraceptives for eight years with two 18-month gaps when she had her children. The agents used included norethisterone-mestranol (Minovlar and Ovlar). In 1971 she presented with a lump in the upper abdomen and investigations suggested that this was a pancreatic cyst. The lump was causing no symptoms and laparotomy was not advised. She remained well, maintaining normal weight and appetite. The lump remained unchanged and in 1974 she was admitted to our unit for further assessment. She had been diagnosed in childhood as suffering from pulmonary siderosis and splenectomy was performed when she was 6 years old. At operation the liver was noted to be normal and a liver biopsy specimen, taken because of the pulmonary siderosis, was reported as normal. She had had a ruptured tubal pregnancy in 1970. She had gluten enteropathy and allergic asthma and was taking disodium cromoglycate and beclomethasone. On 6 February 1974 laparotomy showed a solid tumour 10 by $10 \mathrm{~cm}$ in the left lobe of the liver with a nodular surface. A frozen section of a biopsy specimen was reported as a hamartoma. The biopsy wound was closed to produce haemostasis but no further part of the mass was removed. The remainder of the liver was normal, as also was the pancreas. She recovered rapidly from the procedure and was discharged home well on the tenth day.

Case 3-A 51-year-old woman had taken Gynovlar 21 for eight years. In November 1973 she had a routine cholecystectomy for gall stones and at operation was found to have a mass in the tip of the right lobe of the liver. The gall bladder peeled easily off the surface of the tumour. Biopsy was not attempted and after an uneventful recovery she had two liver scans, one in December 1973 and the other in January 1974. These showed no change in the size of the mass. She was then referred to our unit. Coeliac angiography showed an abnormal circulation in the region of the mass (fig. 1). Pooling of the contrast medium lasted for up to 25 seconds (fig. 2). Though the radiographical appearanoes suggested that the lesion was probably benign a five-day course of intra-arterial chemotherapy was given via the coeliac catheter using $5 \mathrm{~g}$ fluorouracil, $1 \mathrm{~g}$ cyclophosphamide, $2 \mathrm{mg}$ vincristine, and 50 mg methotrexate in divided doses. Two weeks after completing the chemotherapy laparotomy was performed and the tumour was removed by local resection of the tip of the right lobe of the liver. The tumour was $5 \mathrm{~cm}$ in diameter, an oval fleshy mass with a coarsely nodular surface. She made an excellent recovery, returning home 11 days after the operation, and remained well.

\section{Histopathology}

The histological appearances in all three cases were similar. In 


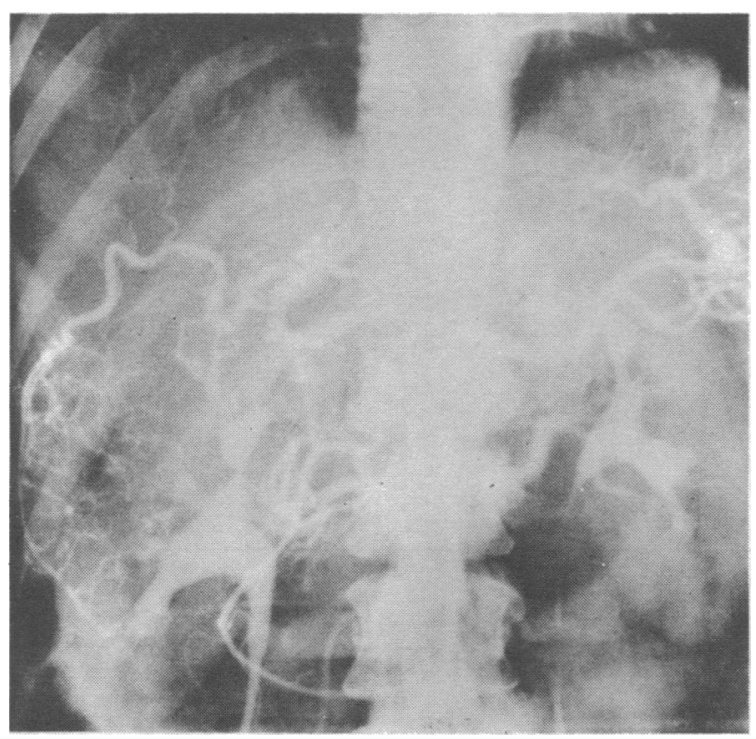

FIG. 1 -Case. 3 Coeliac angiogram. Arterial phase.

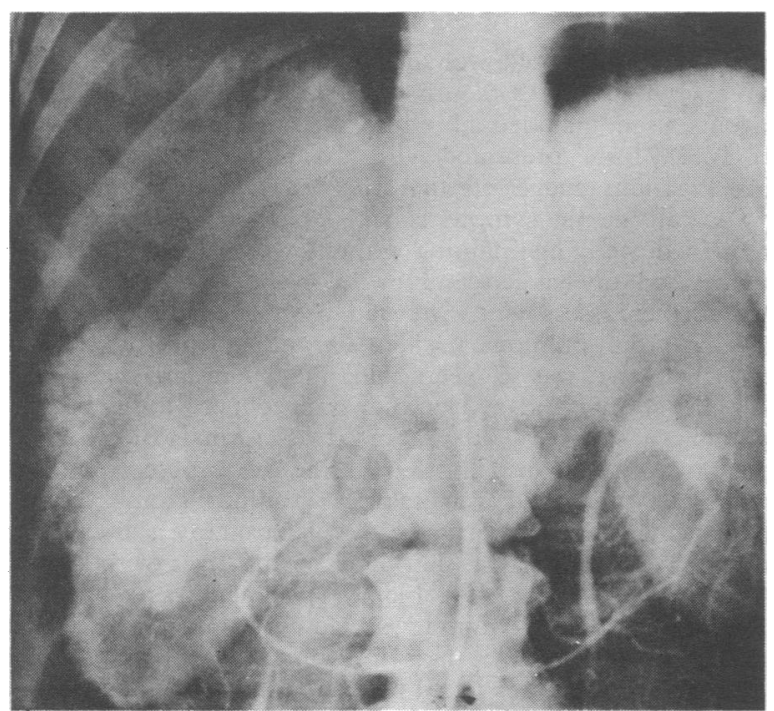

FIG. 2-Case 3. Coeliac angiogram. Late arterial-capillary phase.

each case there was a mass of abnormally arranged liver tissue. This was well circumscribed in all cases but was encapsulated only in case 3. The mass was composed of nodules of liver cell cords, the nodules varying in size and being separated by fibrous trabeculae. There was no evidence of bile retention. The liver cells showed no undue pleomorphism and though some of the hepatocytes were binuclear there was no significant evidence of regenerative or proliferative activity. There was marked proliferation of bile ductular epithelium in the fibrous septa (fig. 3), most striking in case 1, with a focal lymphocytic infiltrate in the portal tracts in cases 1 and 3 . In all three cases there were numerous dilated thin-walled blood vessels lined with endothelium (fig. 4). These were most prominent in cases 1 and 3. In these two cases there were in addition small muscular arteries, one of which, in case 3, contained old organized and recanalized thrombus. Arteries were not present in the material from case 2. Adjacent liver tissue included in the specimens from cases 1 and 3 and a separate specimen from the right lobe of the liver in case 2 showed normal appearances. In particular there was no evidence of cirrhosis in any case.

All three specimens were reported as either hamartomatous

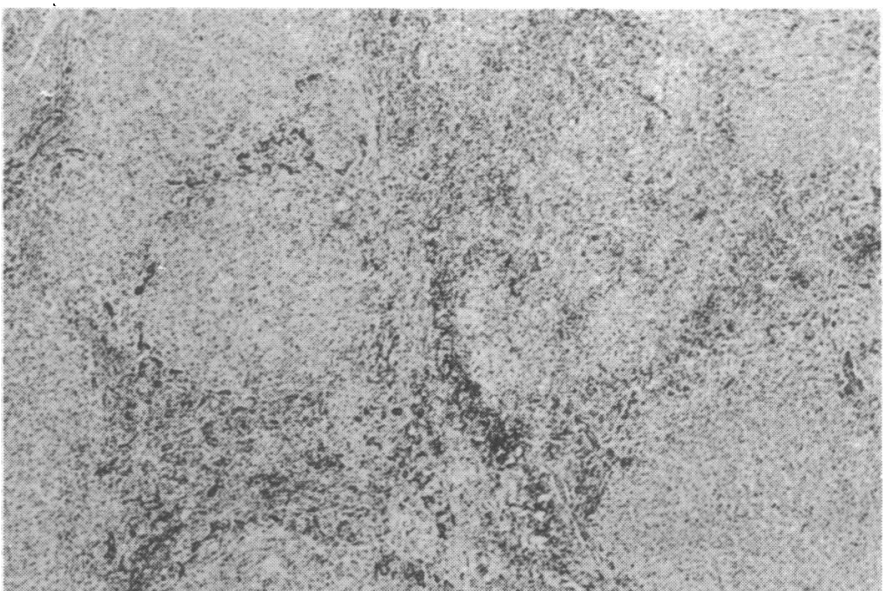

FIG. 3-Case 1. Nodularity and bile ductular proliferation. (Haematoxylin and cosin. $\times 23$ ).

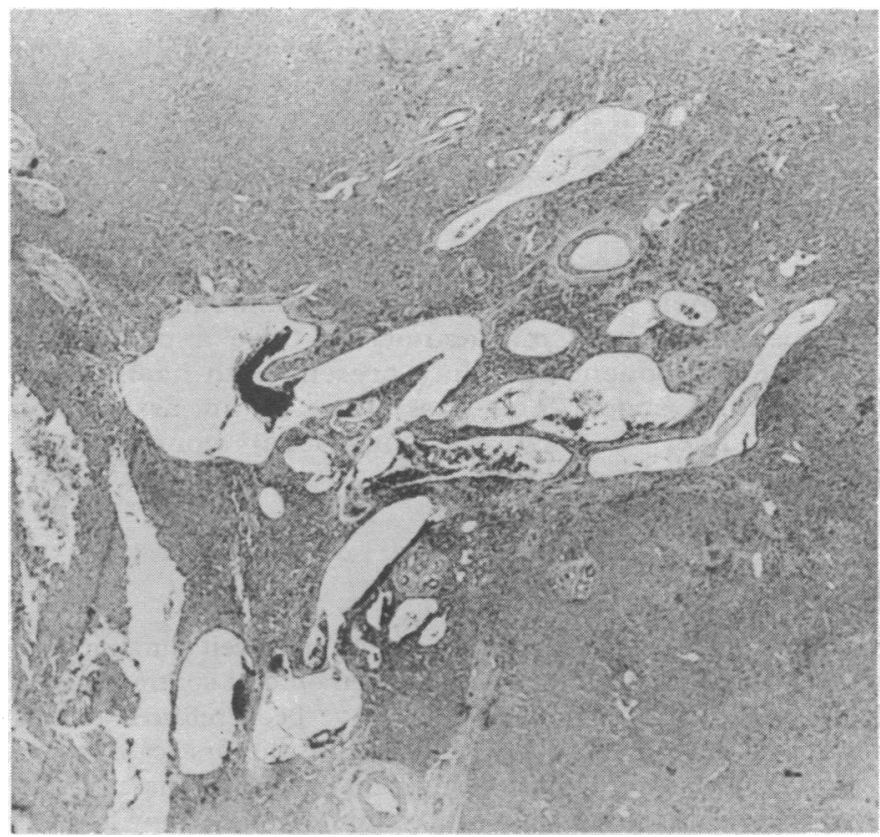
FiG. 4-Case 3. Dilated thin-walled blood ressels. (Haematoxylin and
cosin. $\times 18$ ).

nodule or hepatic malformation. In case 1 benign hepatoma was given as an alternative diagnosis. In the other two cases it was emphasized that though there might be a difference of opinion on the fundamental nature of the lesion it would be expected to behave in a benign manner whether it was a hamartoma or a benign neoplasm.

\section{Discussion}

Since October 1973, 11 cases have been reported of benign liver tumours occurring in women whohad been on oral contraceptives (see table). In the three cases in which angiography was performed the arteriogram showed a well-circumscribed hypervascular mass with an abnormal vasculature suggestive of a benign tumour. In nine cases the patient presented with pain either from haemorrhage into the tumour or from frank rupture with haemoperitoneum. In two cases the patient or her physician felt a lump in the upper abdomen. Local resection or partial 
Details of Women on Oral Contraception who developed Hepatic Lesions

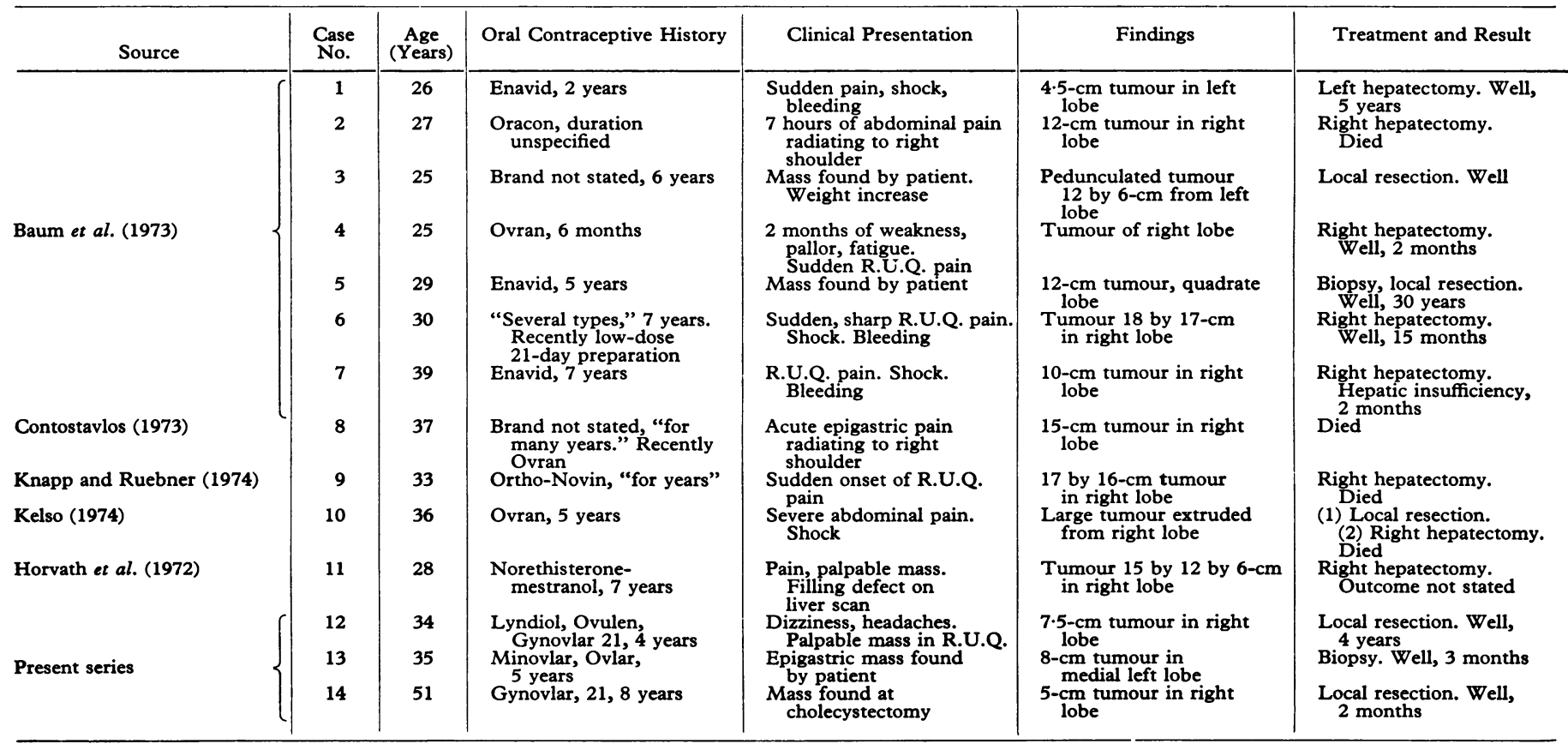

R.U.Q. = Right upper quadrant.

hepatectomy was curative in seven cases. Four patients died, three after massive liver resection and one before admission to hospital. Histologically the tumour in all cases was described as a hepatic adenoma. All the tumours were relatively vascular and in two cases comment was directed towards the presence of large, dilated, blood-filled spaces, so-called "peliosis hepatis."

The three patients reported on here had been on oral contraceptives for periods of at least four years. One patient presented because of vague gastrointestinal symptoms, one because of a symptomless abdominal lump, and in one patient the liver mass was found incidentally at cholecystectomy. Coeliac angiography was performed in two patients and this showed an abnormal vasculature. In these two patients the lesion was at the tip of the right lobe and local removal was performed. In the third patient (case 2) the nodule was in the medial part of the left lobe and only frozen-section biopsy was performed, confirming its benign nature. Thus clinically and radiologically these three cases were similar to the 11 previously reported. Also the macroscopic appearances of the lesions were similar to those in the previous cases.

In our three patients the lesions are best described as hamartomatous nodules-that is, tumour-like malformations in which the various tissues of the part are present in improper proportions or distribution (Willis, 1967)-rather than benign liver cell adenomas. In all cases dilated endothelium-lined vascular channels were present and the remainder of the liver was normal. It is not clear from the previous reports what entity was being described. The lesion was variously diagnosed as a hepatic adenoma, abnormal liver cell hyperplasia with peliosis, hepatic cell adenoma with peliosis, and benign hepatoma. Reference was made to the description of Edmondson (1958), in the A.F.I.P. fascicle, itself a rather diffuse and inconclusive account. It is likely that the differences between our cases and those previously reported are mainly terminological.

The dilated thin-walled vessels which constitute such an important feature of the lesion from the clinical viewpoint have been called "peliosis." Peliosis hepatis refers to two different histological appearances (Yanoff and Rawson, 1964) and it is doubtful whether the term contributes to the description of these lesions. If it is to be used then the peliosis was of the phlebectatic type in our three patients.
Apart from the three patients described here only one other patient with hamartoma of the liver has been seen in this hospital over the past 12 years. This patient was a 66 -year-old man who had a carcinoma of the right adrenal weighing $3.5 \mathrm{~kg}$. At operation to remove this a liver nodule was found and biopsy confirmed its hamartomatous nature. There have been no patients with hepatic adenoma in the hospital during this period. Other authors agree on the rarity of the conditions. There is no discernible pattern in the particular contraceptives that have been taken either in other reports or in the present one.

The Committee on Safety of Medicines' report (1972) showed that there was an increased incidence of liver tumours in rats given certain combinations of steroid hormones. The committee, however, concluded that there was no significant hazard in women taking oral contraceptives. The effect of steroid hormones, and in particular oral contraceptives, on the liver has recently been well and succinctly reviewed (Lancet, 1973). It is interesting that the only other patient with a liver hamartoma in this hospital in recent years should have an adrenal carcinoma. Though this tumour was non-functioning in its clinical manifestations tumours of this nature are known to be capable of performing certain of the steps in corticosteroid biosynthesis, and it is thought that they do produce steroids but that these are biologically inactive (Symington, 1969). If there is a causal relation between oral contraceptive therapy and the development of liver nodules it may operate through the steroid providing a growth stimulus to a pre-existing developmental abnormality.

The entity, though uncommon, is important in that it is potentially fatal. The diagnosis must be considered in any patient who has clinical features of bleeding into the peritoneal cavity, particularly if a mass is palpable in the upper abdomen. One patient (Contostavlos, 1973) was diagnosed as a case of acute cholecystitis and treated with pethidine and oxytetracycline. She died at home three hours later. Both liver scan and coeliac arteriography are helpful in the diagnosis of the nonemergency case, the latter giving more specific information. Needle biospy is contraindicated because of the risk of haemorrhage. Treatment in the emergency case is aimed at controlling blood loss with some form of liver resection. In the elective case the choice is between local removal and a formal lobectomy. 
Where possible the former is to be preferred as the less severe procedure. There is, however, no doubt that these hamartomas should be removed in view of the high mortality rate from haemorrhage in the reported cases. One patient in this series (case 2) was left with a hepatic lesion and only biopsy was performed. At the time of of writing she was being carefully followed up while the situation was reviewed. Her hamartoma was the least vascular in our three cases. She stopped taking oral contraceptives.

All three patients were referred to this hospital from other parts of the country for specialized liver surgery. It is not possible, therefore, to make any inferences about the incidence of hepatic hamartoma whether occurring in patients on oral contraceptives or not.

More cases will have to be described before an association between liver hamartomas and oral contraceptive therapy can be proved. It is surprising that no cases were described until recently and that none have been reported from the United Kingdom until now. In the meantime, however, it seems prudent for practitioners to exclude the presence of an epigastric mass in their patients before prescribing oral contraceptives and also to bear in mind the possibility of hepatic hamartoma or adenoma as a cause of haemoperitoneum in women on the pill.
We wish to thank Mr. Rodney Smith for his advice and for permission to report details of patients under his care, and Professor Sir Theo Crawford for his encouragement and advice on the preparation of the manuscript. We also thank Dr. J. Edmund Williams and the staff of the department of diagnostic radiology, who performed the arteriography, and Miss I. J. Underhill for the preparation of the photographs.

\section{References}

Baun, J. K., et al. (1973). Lancet, 2, 926.

Committee on Safety of Medicines (1972). Carcinogenicity Tests of Oral Contraceptives. London, H.M.S.O.

Contostavlos, D. L. (1973). Lancet, 2, 1200.

Edmondson, H. A. (1958). Tumours of the Liver and Intrahepatic Bile Ducts, p. 18. Washington, D.C. Armed Forces Institute of Pathology.

Horvath, E., Kovacs, K., and Ross, R. C. (1972). Digestion, 7, 74.

Kelso, D. R. (1974), Lancet, 1, 315.

Knapp, W. A., and Ruebner, B. H. (1974). Lancet, 1, 270.

Lancet, 1973, 2, 1281 .

Symington, T. (1969). Functional Pathology of the Human Adrenal Gland, p. 157. Edinburgh, Livingstone.

Willis, R. A. (1967). Pathology of Tumours, 4th edn, p. 7. London Butterworths.

Yanoff, M., and Rawson, A. J. (1964). Archives of Pathology, 77, 159.

\title{
Comparison of Mobilization after Two and Nine Days in Uncomplicated Myocardial Infarction
}

\author{
M. J. HAYES, G. K. MORRIS, J. R. HAMPTON
}

British Medical fournal, 1974, 3, 10-13

\begin{abstract}
Summary
A total of 189 patients with uncomplicated myocardial infarction were selected at random for early or late mobilization and discharge from hospital. Patients were admitted to the study after 48 hours in a coronary care unit if they were free of pain and showed no evidence of heart failure or significant dysrhythmia. Randomization was achieved by monthly cross-over of the three medical wards to which the patients were discharged. One group of patients was mobilized immediately and discharged home after a total of nine days in hospital, and the second group was mobilized on the ninth day and discharged on the 16th day. Outpatient assessment was carried out six weeks after admission. No significant differences were observed between the groups in terms of mortality or morbidity, as reflected by the incidence of recurrent chest pain or myocardial infarction, heart failure, dysrhythmia, or venous thromboembolism detected either clinically or by ${ }^{125}$ Ilabelled fibrinogen scanning.
\end{abstract}

\section{Introduction}

There is still doubt about the optimum period of bed rest and hospital stay for parients with uncomplicated myocandial infarction. Because of the risk of venous thrombosis associ-

Department of Medicine, General Hospital, Nottingham NG1 6HA M. J. HAYES, M.B., M.R.C.P., Research Fellow

G. K. MORRIS, M.B., M.R.C.P., Lecturer in Medicine

J. R. HAMPTON, D.M., M.R.C.P., Consultant Physician and Senior Lecturer in Medicine ated with immobility a short period of "bed rest seems desirable (Gibbs, 1957), and a short stay in hospital might be expected to benefit the patient psychologically (Levine and Lown, 1952). A policy of early discharge from hospital will also help to relieve pressure on beds for acutely ill patients.

As most deaths and complications from myocardial infarction occur within 48 hours of the onset of symptoms (Spracklen et al., 1968; Fulton et al., 1969) it seems reasonable that the period of hospital stay should be quite short. Several studies have compared the effect of varying periods of bed rest and hospital stay in patients with uncomplicated myocardial infarction (Harpur et al., 1971; Hutter et al., 1973; Medical Division, Royal Infirmary, Glasgow, 1973), and despite a progressive reduction in the time spent in bed and in hospital no difference between patients mobilized "early" or "late" has been observed in terms of morbidity or mortality. Here we describe a randomized trial of mobilization at 48 hours and discharge home after nine days compared with mobilization at nine days and discharge after 16 days in hospital.

\section{Patients and Methods}

Patients who had been admitted to the coronary care unit (C.C.U.) at Nottingham General Hospital because of a suspected myocardial infarction within the preceding 24 hours were considered for inclusion in the trial. All such patients had a history suggestive of myocardial infarction and the diagnosis was accepted if either characteristic E.C.G. changes or a rise in the serum enzymes (creatinine phosphokinase, aspartate aminotransferase (SGOT), lactic dehydrogenase, or hydroxybutyric dehydrogenase) were shown. All patients who survived 48 hours after admission to hospital were examined by a member of the study team. They were considered unfit for mobilization, and therefore ex- 